\title{
Partial Derivatives of Three Variables Functions
}

\author{
Chii-Huei Yu \\ Department of Management and Information, Nan Jeon University of Science and Technology, Tainan City, 73746, Taiwan \\ *Corresponding Author: chiihuei@mail.nju.edu.tw
}

Copyright $@ 2014$ Horizon Research Publishing All rights reserved.

\begin{abstract}
This paper takes the mathematical software Maple as the auxiliary tool to study the partial differential problem of two types of three variables functions. We can obtain the infinite series forms of any order partial derivatives of these two types of functions by using differentiation term by term theorem, and hence greatly reduce the difficulty of calculating higher order partial derivative values of these functions. On the other hand, we propose two examples to do calculation practically.
\end{abstract}

Keywords Partial Derivatives, Infinite Series Forms, Differentiation Term By Term Theorem, Maple

\section{Introduction}

In calculus and engineering mathematics curricula, evaluating the $n$-th order partial derivative value of a multivariable function at some point, in general, needs to go through two procedures: firstly determining the $n$-th order partial derivative of this function, and then taking the point into this $n$-th order partial derivative. These two procedures will make us face with increasingly complex calculations when calculating higher order partial derivative values ( i.e. $n$ is large), and hence to obtain the answers by manual calculations is not easy. In this paper, we study the partial differential problem of the following two types of three variables functions

$$
\begin{gathered}
f(x, y, t)=\ln \left[e^{2 a x}-2 e^{a x} y^{b} \cos (c t+d)+y^{2 b}\right] \\
g(x, y, t)=\tan ^{-1}\left[\frac{y^{b} \sin (c t+d)}{e^{a x}-y^{b} \cos (c t+d)}\right]
\end{gathered}
$$

Where $a, b, c, d$ are real numbers. We can obtain the infinite series forms of any order partial derivatives of these two types of functions by using differentiation term by term theorem ; these are the major results of this study (i.e., Theorems 1 and 2), and hence greatly reduce the difficulty of calculating their higher order partial derivative values. For the study of related partial differential problems can refer to [1-12]. In addition, we provide two examples to do calculation practically. The research methods adopted in this study involved finding solutions through manual calculations and verifying these solutions by using Maple. This type of research method not only allows the discovery of calculation errors, but also helps modify the original directions of thinking from manual and Maple calculations. Therefore, Maple provides insights and guidance regarding problem-solving methods.

\section{Main Results}

Firstly, we introduce a definition and some notations, formulas used in this paper.

\subsection{Definition}

The complex natural logarithm function $\ln z$ is defined by $\ln z=\ln |z|+i \theta$, where $z$ is a complex number, $\theta$ is a real number, and $z=|z| \cdot e^{i \theta},-\pi \leq \theta<\pi$.

\subsection{Notations}

2.2.1. Suppose $r$ is any real number, $m$ is any positive integer. Define $(r)_{m}=r(r-1) \cdots(r-m+1)$, and $(r)_{0}=1$. 2.2.2. Let $z=a+i b$ be a complex number, where $i=\sqrt{-1}, \quad a, b$ are real numbers. We denote $a$ the real part of $z$ by $\operatorname{Re}(z)$, and $b$ the imaginary part of $z$ by $\operatorname{Im}(z)$.

2.2.3. Assume $p, q, r$ are non-negative integers. For the three variables function $f(x, y, t)$, the $p$ times partial derivative with respect to $x, q$ times partial derivative with respect to $y$, and $r$ times partial derivative with respect to $t$, forms a $p+q+r$-th order partial derivative of $f(x, y, t)$, and denoted by

$$
\frac{\partial^{p+q+r} f}{\partial t^{r} \partial y^{q} \partial x^{p}}(x, y, t)
$$

\subsection{Formulas}

\subsection{Euler's formula}


$e^{i \alpha}=\cos \alpha+i \sin \alpha$, where $\alpha$ is any real number.

\subsubsection{DeMoivre's Formula}

$(\cos \alpha+i \sin \alpha)^{n}=\cos n \alpha+i \sin n \alpha$, where $n$ is any integer, and $\alpha$ is any real number.

2.3.3. $\ln (1-z)=-\sum_{k=1}^{\infty} \frac{1}{k} z^{k}$, where $z$ is a complex number, $|z|<1$.

Next, we introduce an important theorem used in this study.

\subsection{Differentiation term by term theorem ([13])}

If, for all non-negative integer $k$, the functions $g_{k}:(a, b) \rightarrow R$ satisfy the following three conditions : (i) there exists a point $x_{0} \in(a, b)$ such that $\sum_{k=0}^{\infty} g_{k}\left(x_{0}\right)$ is convergent, (ii) all functions $g_{k}(x)$ are differentiable on open interval $(a, b)$, (iii) $\sum_{k=0}^{\infty} \frac{d}{d x} g_{k}(x)$ is uniformly convergent on $(a, b)$. Then $\sum_{k=0}^{\infty} g_{k}(x)$ is uniformly convergent and differentiable on $(a, b)$. Moreover, its derivative $\frac{d}{d x} \sum_{k=0}^{\infty} g_{k}(x)=\sum_{k=0}^{\infty} \frac{d}{d x} g_{k}(x)$.

Before deriving the major results in this study, we need a lemma.

2.5. Lemma A Suppose $\lambda, \alpha$ are real numbers, $|\lambda|<1$. Then

$$
\begin{aligned}
& \ln \left(1-\lambda e^{i \alpha}\right) \\
& =\frac{1}{2} \ln \left(1-2 \lambda \cos \alpha+\lambda^{2}\right)-i \tan ^{-1}\left(\frac{\lambda \sin \alpha}{1-\lambda \cos \alpha}\right)
\end{aligned}
$$

\subsubsection{Proof}

$$
\begin{aligned}
& \ln \left(1-\lambda e^{i \alpha}\right) \\
& =\ln (1-\lambda \cos \alpha-i \lambda \sin \alpha) \quad(\text { By Euler's formula }) \\
& =\ln \left[\sqrt{(1-\lambda \cos \alpha)^{2}+(\lambda \sin \alpha)^{2}} \times\right. \\
& \left.\left(\frac{1-\lambda \cos \alpha}{\sqrt{(1-\lambda \cos \alpha)^{2}+(\lambda \sin \alpha)^{2}}}-i \frac{\lambda \sin \alpha}{\sqrt{(1-\lambda \cos \alpha)^{2}+(\lambda \sin \alpha)^{2}}}\right)\right] \\
& =\ln \left[\sqrt{(1-\lambda \cos \alpha)^{2}+(\lambda \sin \alpha)^{2}}\right]+
\end{aligned}
$$

$$
\ln \left[\frac{1-\lambda \cos \alpha}{\sqrt{(1-\lambda \cos \alpha)^{2}+(\lambda \sin \alpha)^{2}}}-i \frac{\lambda \sin \alpha}{\sqrt{(1-\lambda \cos \alpha)^{2}+(\lambda \sin \alpha)^{2}}}\right]
$$$$
=\ln \left[\sqrt{1-2 \lambda \cos \alpha+\lambda^{2}}\right]+
$$

$$
\ln \left[\frac{1-\lambda \cos \alpha}{\sqrt{1-2 \lambda \cos \alpha+\lambda^{2}}}-i \frac{\lambda \sin \alpha}{\sqrt{1-2 \lambda \cos \alpha+\lambda^{2}}}\right]
$$$$
=\frac{1}{2} \ln \left(1-2 \lambda \cos \alpha+\lambda^{2}\right)-i \tan ^{-1}\left(\frac{\lambda \sin \alpha}{1-\lambda \cos \alpha}\right)
$$

\section{( By Definition 2.1)}

The following is the first major result in this study, we determine the infinite series forms of any order partial derivatives of the three variables function (1).

\subsection{Theorem 1}

Let $a, b, c, d$ be real numbers, $p, q, r$ be non-negative integers. Suppose the domain of the three variables function

$$
f(x, y, t)=\ln \left[e^{2 a x}-2 e^{a x} y^{b} \cos (c t+d)+y^{2 b}\right]
$$

is $\left\{(x, y, t) \in R^{3} \mid y^{b}\right.$ exists, $\left.y \neq 0,\left|y^{b}\right|<e^{a x}\right\}$.

Then the $p+q+r$-th order partial derivative of $f(x, y, t)$,

$$
\begin{gathered}
\frac{\partial^{p+q+r} f}{\partial t^{r} \partial y^{q} \partial x^{p}}(x, y, t)=2 a \cdot(1)_{p} x^{1-p}-2(-1)^{p} a^{p} c^{r} \times \\
\sum_{k=1}^{\infty} k^{p+r-1}(b k)_{q} e^{-a k x} y^{b k-q} \cos \left[k(c t+d)+\frac{r \pi}{2}\right](4)
\end{gathered}
$$

\subsubsection{Proof}

Let $\lambda, \alpha$ be real numbers, $|\lambda|<1$.

Because

$$
\begin{aligned}
& \frac{1}{2} \ln \left(1-2 \lambda \cos \alpha+\lambda^{2}\right) \\
& =\operatorname{Re}\left[\ln \left(1-\lambda e^{i \alpha}\right)\right] \quad(\text { By Lemma A) } \\
& =\operatorname{Re}\left[-\sum_{k=1}^{\infty} \frac{1}{k}\left(\lambda e^{i \alpha}\right)^{k}\right] \quad \text { (By Formula 2.3.3) } \\
& =\operatorname{Re}\left[-\sum_{k=1}^{\infty} \frac{1}{k} \lambda^{k} e^{i k \alpha}\right] \quad \text { (By DeMoivre's formula) } \\
& =-\sum_{k=1}^{\infty} \frac{1}{k} \lambda^{k} \cos k \alpha \quad \text { (By Euler's formula) }
\end{aligned}
$$


Taking $\lambda=\frac{y^{b}}{e^{a x}}, \alpha=c t+d$ into (5), we obtain

$f(x, y, t)$

$$
\begin{aligned}
& =\ln \left[e^{2 a x}-2 e^{a x} y^{b} \cos (c t+d)+y^{2 b}\right] \\
& =2 a x-2 \sum_{k=1}^{\infty} \frac{1}{k} e^{-a k x} y^{b k} \cos k(c t+d)
\end{aligned}
$$

Thus, by differentiation term by term theorem, differentiating $p$ times with respect to $x, q$ times with respect to $y$, and $r$ times with respect to $t$ on both sides of (6), we obtain the $p+q+r$-th order partial derivative of $f(x, y, t)$,

$$
\begin{aligned}
& \frac{\partial^{p+q+r} f}{\partial t^{r} \partial y^{q} \partial x^{p}}(x, y, t)=2 a \cdot(1)_{p} x^{1-p}-2(-1)^{p} a^{p} c^{r} \times \\
& \sum_{k=1}^{\infty} k^{p+r-1}(b k)_{q} e^{-a k x} y^{b k-q} \cos \left[k(c t+d)+\frac{r \pi}{2}\right]
\end{aligned}
$$

The following is the second major result in this paper, we obtain the infinite series forms of any order partial derivatives of the three variables function (2).

\subsection{Theorem 2}

If the assumptions are the same as

Theorem 1. Suppose the domain of the three variables function

$$
g(x, y, t)=\tan ^{-1}\left[\frac{y^{b} \sin (c t+d)}{e^{a x}-y^{b} \cos (c t+d)}\right]
$$

is

$$
\left\{(x, y, t) \in R^{3} \mid y^{b} \text { exists, } y \neq 0,\left|y^{b}\right|<e^{a x}\right\} .
$$

Then the $p+q+r$-th order partial derivative of $g(x, y, t)$,

$$
\begin{gathered}
\frac{\partial^{p+q+r} g}{\partial t^{r} \partial y^{q} \partial x^{p}}(x, y, t)=(-1)^{p} a^{p} c^{r} \times \\
\sum_{k=1}^{\infty} k^{p+r-1}(b k)_{q} e^{-a k x} y^{b k-q} \sin \left[k(c t+d)+\frac{r \pi}{2}\right]
\end{gathered}
$$

\subsubsection{Proof}

Let $\lambda, \alpha$ be real numbers, $|\lambda|<1$.

Because

$$
\tan ^{-1}\left(\frac{\lambda \sin \alpha}{1-\lambda \cos \alpha}\right)
$$

$$
\begin{gathered}
=-\operatorname{Im}\left[\ln \left(1-\lambda e^{i \alpha}\right)\right] \quad(\text { By Lemma A }) \\
=\operatorname{Im}\left[\sum_{k=1}^{\infty} \frac{1}{k}\left(\lambda e^{i \alpha}\right)^{k}\right] \quad(\text { By Formula 2.3.3) } \\
=\operatorname{Im}\left[\sum_{k=1}^{\infty} \frac{1}{k} \lambda^{k} e^{i k \alpha}\right] \quad(\text { By DeMoivre's formula }) \\
=\sum_{k=1}^{\infty} \frac{1}{k} \lambda^{k} \sin k \alpha \quad \text { (By Euler's formula) }
\end{gathered}
$$

Taking $\lambda=\frac{y^{b}}{e^{a x}}, \alpha=c t+d$ into (8), we obtain

$$
\begin{gathered}
g(x, y, t) \\
=\tan ^{-1}\left[\frac{y^{b} \sin (c t+d)}{e^{a x}-y^{b} \cos (c t+d)}\right] \\
=\sum_{k=1}^{\infty} \frac{1}{k} e^{-a k x} y^{b k} \sin k(c t+d)
\end{gathered}
$$

Thus, using differentiation term by term theorem, differentiating $p$ times with respect to $x, q$ times with respect to $y$, and $r$ times with respect to $t$ on both sides of (9), we obtain the $p+q+r$-th order partial derivative of $g(x, y, t)$,

$$
\begin{array}{r}
\frac{\partial^{p+q+r} g}{\partial t^{r} \partial y^{q} \partial x^{p}}(x, y, t)=(-1)^{p} a^{p} c^{r} \times \\
\sum_{k=1}^{\infty} k^{p+r-1}(b k)_{q} e^{-a k x} y^{b k-q} \sin \left[k(c t+d)+\frac{r \pi}{2}\right]
\end{array}
$$

\section{Examples}

In the following, for the partial differential problem of the two types of three variables functions in this study, we provide two examples and use Theorems 1, 2 to determine the infinite series forms of any order partial derivatives and some higher order partial derivative values of these functions. On the other hand, we employ Maple to calculate the approximations of these higher order partial derivative values and their infinite series forms for verifying our answers.

\subsection{Example 1}

Suppose the domain of the three variables function

$$
f(x, y, t)=\ln \left[e^{8 x}-2 e^{4 x} y^{7 / 3} \cos \left(5 t+\frac{\pi}{4}\right)+y^{14 / 3}\right]
$$

is $\left\{(x, y, t) \in R^{3}|y \neq 0,| y^{7 / 3} \mid<e^{4 x}\right\}$ (the case of 
$a=4, b=\frac{7}{3}, c=5, d=\frac{\pi}{4} \quad$ in Theorem 1)

Using Theorem 1 , we obtain any $p+q+r$-th order partial derivative of $f(x, y, t)$,

$$
\begin{gathered}
\frac{\partial^{p+q+r} f}{\partial t^{r} \partial y^{q} \partial x^{p}}(x, y, t)=8 \cdot(1)_{p} x^{1-p}-2(-1)^{p} 4^{p} 5^{r} \times \\
\sum_{k=1}^{\infty} k^{p+r-1}\left(\frac{7 k}{3}\right)_{q} e^{-4 k x} y^{7 k / 3-q} \cos \left[k\left(5 t+\frac{\pi}{4}\right)+\frac{r \pi}{2}\right]
\end{gathered}
$$

Therefore, we can determine the 11-th order partial derivative value of $f(x, y, t)$ at $\left(2,3, \frac{\pi}{4}\right)$

$$
\begin{gathered}
\frac{\partial^{11} f}{\partial t^{4} \partial y^{3} \partial x^{4}}\left(2,3, \frac{\pi}{4}\right) \\
=-2 \cdot 4^{4} \cdot 5^{4} \cdot \sum_{k=1}^{\infty} k^{7}\left(\frac{7 k}{3}\right)_{3} e^{-8 k} \cdot 3^{7 k / 3-3} \cdot \cos \left(\frac{k \pi}{2}\right)
\end{gathered}
$$

Next, we use Maple to verify the correctness of (12).

$>\mathrm{f}:=(\mathrm{x}, \mathrm{y}, \mathrm{t})->\ln \left(\exp \left(8^{*} \mathrm{x}\right)-2 * \exp (4 * \mathrm{x})\right)^{*} \mathrm{y}^{\wedge}(7 / 3) * \cos (5 * \mathrm{t}+\mathrm{Pi} / 4)$ $\left.+\mathrm{y}^{\wedge}(14 / 3)\right)$;

$f:=(x, y, t) \rightarrow \ln \left(\mathrm{e}^{8 x}-2 \mathrm{e}^{4 x} y^{7 / 3} \cos \left(5 t+\frac{1}{4} \pi\right)+y^{14 / 3}\right)$

$>$ evalf(D[1\$4,2\$3,3\$4](f)(2,3,Pi/4),26);

1272.7259478530745929831110

$>$ evalf $\left(-2 * 4^{\wedge} 4 * 5^{\wedge} 4 * \operatorname{sum}\left(\mathrm{k}^{\wedge} 7^{*} \operatorname{product}\left(7^{*} \mathrm{k} / 3-\mathrm{j}, \mathrm{j}=0 . .2\right) * \operatorname{ex}\right.\right.$ $\mathrm{p}\left(-8^{*} \mathrm{k}\right) * 3^{\wedge}\left(7^{*} \mathrm{k} / 3-3\right) * \cos (\mathrm{k} * \mathrm{Pi} / 2), \mathrm{k}=1$.. infinity $\left.), 26\right)$;

1272.7259478530745929830877

\subsection{Example 2}

Suppose the domain of the three variables function

$$
g(x, y, t)=\tan ^{-1}\left[\frac{y^{9 / 4} \sin \left(3 t-\frac{5 \pi}{6}\right)}{e^{-6 x}-y^{9 / 4} \cos \left(3 t-\frac{5 \pi}{6}\right)}\right]
$$

is

$$
\left\{(x, y, t) \in R^{3}|y>0,| y^{9 / 4} \mid<e^{-6 x}\right\}
$$

(the case of $a=-6, b=\frac{9}{4}, c=3, d=-\frac{5 \pi}{6}$ in Theorem 2)

By Theorem 2, we obtain any $p+q+r$-th order partial derivative of $g(x, y, t)$,

$$
\frac{\partial^{p+q+r} g}{\partial t^{r} \partial y^{q} \partial x^{p}}(x, y, t)=6^{p} \cdot 3^{r} \times
$$

$$
\sum_{k=1}^{\infty} k^{p+r-1}\left(\frac{9 k}{4}\right)_{q} e^{6 k x} y^{9 k / 4-q} \sin \left[k\left(3 t-\frac{5 \pi}{6}\right)+\frac{r \pi}{2}\right]
$$

Thus, we can evaluate the 13-th order partial derivative value of $g(x, y, t)$ at $\left(-2,7,-\frac{5 \pi}{6}\right)$

$$
\begin{gathered}
\frac{\partial^{13} g}{\partial t^{4} \partial y^{6} \partial x^{3}}\left(-2,7,-\frac{5 \pi}{6}\right) \\
=6^{3} \cdot 3^{4} \cdot \sum_{k=1}^{\infty} k^{6}\left(\frac{9 k}{4}\right)_{6} e^{-12 k} 7^{9 k / 4-6} \sin \left(\frac{2 k \pi}{3}\right)
\end{gathered}
$$

We also use Maple to verify the correctness of (15). $>\mathrm{g}:=(\mathrm{x}, \mathrm{y}, \mathrm{t})->\arctan \left(\left(\mathrm{y}^{\wedge}(9 / 4) * \sin (3 * \mathrm{t}-5 * \mathrm{Pi} / 6)\right) /(\exp (-6 * \mathrm{x})-\right.$ $\left.\left.\mathrm{y}^{\wedge}(9 / 4) * \cos (3 * \mathrm{t}-5 * \mathrm{Pi} / 6)\right)\right)$;

$$
g:=(x, y, t) \rightarrow \arctan \left(\frac{y^{9 / 4} \sin \left(3 t-\frac{5}{6} \pi\right)}{\mathrm{e}^{-6 x}-y^{9 / 4} \cos \left(3 t-\frac{5}{6} \pi\right)}\right)
$$

$>$ evalf(D[1\$3,2\$6,3\$4](g)(-2,7,-5*Pi/6), 18);

$$
-0.000129060832915015486
$$

$>\operatorname{evalf}\left(6^{\wedge} 3^{*} 3^{\wedge} 4 * \operatorname{sum}\left(\mathrm{k}^{\wedge} 6^{*} \operatorname{product}(9 * \mathrm{k} / 4-\mathrm{j}, \mathrm{j}=0 . .5) * \exp (-1\right.\right.$ $2 * \mathrm{k}) * 7^{\wedge}(9 * \mathrm{k} / 4-6) * \sin (2 * \mathrm{k} * \mathrm{Pi} / 3), \mathrm{k}=1$...infinity $\left.), 18\right)$;

\section{$-0.000129060832915015502$}

\section{Conclusion}

As mentioned, the differentiation term by term theorem plays a significant role in the theoretical inferences of this study. In fact, the application of this theorem is extensive, and can be used to easily solve many difficult problems; we endeavor to conduct further studies on related applications. On the other hand, Maple also plays a vital assistive role in problem-solving. In the future, we will extend the research topic to other calculus and engineering mathematics problems and solve these problems by using Maple. These results will be used as teaching materials for Maple on education and research to enhance the connotations of calculus and engineering mathematics.

\section{REFERENCES}

[1] A. Griewank and A. Walther, Evaluating Derivatives: Principles and Techniques of Algorithmic Differentiation, 2nd ed., SIAM, Philadelphia, 2008.

[2] D. N. Richard, An efficient method for the numerical evaluation of partial derivatives of arbitrary order, ACM Transactions on Mathematical Software, Vol. 18, No. 2, pp. 
159-173, 1992.

[3] C. H., Bischof, G. Corliss, and A. Griewank, Structured second and higher-order derivatives through univariate Taylor series, Optimization Methods and Software, Vol. 2, pp. 211-232, 1993.

[4] L. E. Fraenkel, Formulae for high derivatives of composite functions, Mathematical Proceedings of the Cambridge Philosophical Society, Vol. 83, pp. 159-165, 1978.

[5] T-W, Ma, Higher chain formula proved by combinatorics, The Electronic Journal of Combinatorics, Vol. 16, \#N21, 2009.

[6] C. -H. Yu, Partial derivatives of some types of two-variables functions, Pure and Applied Mathematics Journal, Vol. 2, No. 2, pp. 56-61, 2013.

[7] C. -H. Yu, Using Maple to evaluate the partial derivatives of two-variables functions, International Journal of Computer Science and Mobile Computing, Vol. 2, Issue. 6, pp. 225-232, 2013.

[8] C.-H. Yu, Using Maple to study the partial differential problems, Applied Mechanics and Materials, in press.

[9] C. -H. Yu, Evaluating partial derivatives of two-variables functions by using Maple, Proceedings of the 6th IEEE/International Conference on Advanced Infocomm Technology, Taiwan, No. 00295, 2013.

[10] C.-H. Yu, Application of Maple: taking the partial differential problem of some types of two-variables functions as an example, Proceedings of the International Conference on e-Learning, Taiwan, pp. 337-345, 2013.

[11] C.-H. Yu, Application of Maple on the partial differential problem of four types of two-variables functions, Proceedings of the International Conference on Advanced Information Technologies, Taiwan, No.87, 2013.

[12] C.-H., Yu, Application of Maple: taking the partial differential problem of two-variables functions as an example, Proceedings of 2013 Business Innovation and Development Symposium, Taiwan, B20130113001, 2013.

[13] T. M. Apostol, Mathematical Analysis, 2nd ed., Boston: Addison-Wesley, p230, 1975. 